\title{
Roles of SETD2 in Leukemia-Transcription, DNA-Damage, and Beyond
}

\author{
Anna Skucha ${ }^{1}$, Jessica Ebner ${ }^{2}$ and Florian Grebien ${ }^{2,3, *}$ (i) \\ 1 CeMM-Research Center for Molecular Medicine of the Austrian Academy of Sciences, Lazarettgasse 14, \\ AKH BT 25.3, 1090 Vienna, Austria; an.skucha@gmail.com \\ 2 Ludwig Boltzmann Institute for Cancer Research, Waehringer Strasse 13A, 1090 Vienna, Austria; \\ Jessica.Ebner@lbicr.lbg.ac.at \\ 3 Institute for Medical Biochemistry, University of Veterinary Medicine Vienna, Veterinaerplatz 1, \\ 1210 Vienna, Austria \\ * Correspondence: Florian.Grebien@vetmeduni.ac.at; Tel.: +43-1-25077-4200
}

Received: 17 January 2019; Accepted: 20 February 2019; Published: 27 February 2019

\begin{abstract}
The non-redundant histone methyltransferase SETD2 (SET domain containing 2; KMT3A) is responsible for tri-methylation of lysine 36 on histone $\mathrm{H} 3$ (H3K36me3). Presence of the H3K36me3 histone mark across the genome has been correlated with transcriptional activation and elongation, but also with the regulation of DNA mismatch repair, homologous recombination and alternative splicing. The role of SETD2 and the H3K36me3 histone mark in cancer is controversial. SETD2 is lost or mutated in various cancers, supporting a tumor suppressive role of the protein. Alterations in the SETD2 gene are also present in leukemia patients, where they are associated with aggressive disease and relapse. In line, heterozygous SETD2 loss caused chemotherapy resistance in leukemia cell lines and mouse models. In contrast, other studies indicate that SETD2 is critically required for the proliferation of leukemia cells. Thus, although studies of SETD2-dependent processes in cancer have contributed to a better understanding of the SETD2-H3K36me3 axis, many open questions remain regarding its specific role in leukemia. Here, we review the current literature about critical functions of SETD2 in the context of hematopoietic malignancies.
\end{abstract}

Keywords: SETD2; acute myeloid leukemia; mutations; histone modifications

\section{Introduction}

Histone tails are subject to extensive posttranslational modification, such as methylation, acetylation, phosphorylation, ubiquitination and others, resulting in the establishment of the so-called "histone code". The information encoded in the epigenetic modification of histones is critical for the regulation of a multitude of cellular processes, including transcription, DNA replication and DNA repair after damage. Thus, enzymes modulating these epigenetic processes play an important role in all stages of development and their dysregulation often leads to genetic disorders, including cancer. While the majority of histone modifications are associated with transcriptional control and related processes, the importance and functional consequences of many types of histone modifications remain incompletely understood. This is illustrated by the example of tri-methylation of histone $\mathrm{H} 3$ on lysine 36 (H3K36me3) by the methyltransferase SETD2. H3K36 tri-methylation by SETD2 has been linked to a plethora of critical pathways and processes, yet the functional implications of perturbed H3K36 tri-methylation by SETD2 mutations in cancer are still unclear. In this review, we will discuss the main functions of SETD2 and H3K36 tri-methylation and potential consequences of their dysregulation in hematopoietic malignancies. 
SETD2 was first described in 1998 as a gene associated with Huntington disease [1]. Soon after, it was found to be expressed in human CD34+ hematopoietic stem and progenitor cells and to possess H3K36-specific histone methyltransferase activity [2]. Interestingly, mono- and di-methylation of H3K36 can be catalyzed by a number of promiscuous enzymes, including NSD1, NSD2 and NSD3 (Nuclear receptor-binding SET domain-containing proteins 1-3), ASH1L (Absent small and homeotic disks protein 1 homolog) and SMYD2 (SET and MYND domain containing 2), while SETD2 is the sole methyltransferase responsible for cellular H3K36 tri-methylation, using H3K36me2 as a substrate [3-5].

In general, all functional domains of SETD2 are highly conserved from yeast to humans [6,7]. Set2, the Saccharomyces cerevisiae homologue of the human SETD2 protein is responsible for all three steps of H3K36 methylation [8,9]. The catalytic SET (Suppressor of Variegation, Enhancer of zeste and Trithorax) domain is flanked an AWS (associated with SET) and a Post-SET domain. In addition, SETD2 contains a coiled-coiled (CC) and a WW (Tryptophan-Tryptophan) domain, which are mediating protein-protein interactions [6,7]. Via its functional SR1 (Set2 Rpb1 interacting) domain, SETD2 interacts with the hyper-phosphorylated C-terminal domain (CTD) of Rpb1, the largest subunit of RNA Pol II [10]. The N-terminus of human SETD2 comprises more than half of the protein sequence, but it is not evolutionarily conserved and it is not known if it harbors any functional domains $[8,9]$.

Constitutive knockout of Setd2 resulted in embryonic lethality at E10.5-E11.5 due to defects in the vascular architecture [11]. Setd2 deficiency in the hematopoietic system led to altered differentiation capacity of hematopoietic stem cells. Setd2-knockout mice showed reduced numbers of myeloid, lymphoid and megakaryocyte progenitor cells as well as a strong reduction in phenotypic and functional hematopoietic stem cells [12]. Setd2-deficient hematopoietic progenitors displayed elevated levels of replicative stress, which impairs their proliferative capacity. Consequently, Setd2-deficient hematopoietic progenitors exhibited increased genomic instability, accumulation of secondary mutations and malignant transformation [13]. In addition, hematopoietic stem cells isolated from Setd2-deficient mice displayed elevated rates of apoptosis, reduced multi-potent differentiation potential and consequently differentiated towards mature cell types [12].

\section{SETD2 Mutations in Hematological Malignancies}

Mutations in the SETD2 gene have been described in various human malignancies. Initially, SETD2-inactivating mutations have been found in $15 \%$ of patients with clear cell renal cell carcinoma (ccRCC) [14-16]. Alterations of SETD2 were also identified in $30 \%$ of pediatric high-grade gliomas (HGGs) and colorectal cancer $[17,18]$. Mutations of SETD2 were also found to be associated with hematopoietic malignances (Figure 1). In this cancer entity, mainly missense mutations can be found, which occur across the entire coding sequence. Focal deletions of SETD2 were identified in $10 \%$ of patients suffering from early T-cell precursor acute lymphoblastic leukemia (ETP-ALL) [19]. Bi-allelic loss of SETD2 was identified in mast cell leukemia (MCL) [20]. Moreover, SETD2 mutations have been frequently identified in patients suffering from enteropathy-associated T-cell lymphoma and chronic lymphoblastic leukemia [21,22]. Finally, alterations in the SETD2 gene were significantly enriched in relapsed pediatric acute lymphoblastic leukemia (ALL) patients, pointing towards a potential role of SETD2 mutations in chemotherapy resistance [23]. This was recently confirmed, as heterozygous loss of SETD2 in leukemia resulted in resistance to DNA-damaging agents [24]. These findings and the high prevalence of SETD2 mutations across different cancer entities strongly implied tumor suppressive functions of SETD2 and the corresponding H3K36me3 histone mark in cancer.

Several reports have characterized the role of normal and mutated SETD2 in leukemia with MLL (Mixed Lineage Leukemia)-fusion genes. Zhu et al. described nonsense and frameshift mutations in SETD2 in pediatric patients with MLL-rearrangements [25]. shRNA-mediated knockdown of SETD2 led to proliferative advantage, increased colony formation and accelerated leukemia development of fusion-protein expressing leukemia cells in vitro and in vivo, further establishing a tumor suppressive role of SETD2 in leukemia. Conversely, several genome-scale CRISPR (Clustered Regularly Interspaced Short Palindromic Repeats)/Cas9 screens identified SETD2 as an essential gene in leukemia cells, 
proposing alternative functions of SETD2 in addition to its tumor suppressor role [26-29]. Using a domain-focused CRISPR/Cas9 mutagenesis approach, it was shown that the catalytic activity of SETD2 was essential, as mutagenesis of the SETD2 SET domain impaired the proliferation of MLL-AF9-expressing leukemia cells [29]. In line with this, we recently found that shRNA- and CRISPR/Cas9-mediated loss of SETD2 led to differentiation, enhanced DNA damage and apoptosis of acute myeloid leukemia (AML) cells harboring MLL-fusions in vitro and in vivo [30].

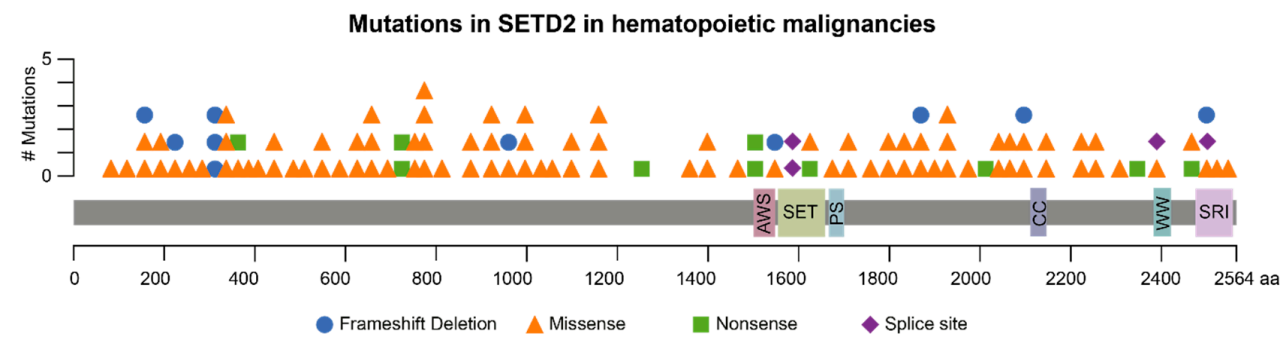

Figure 1. Schematic representation of SETD2 mutations associated with hematopoietic malignancies. Mutations are represented according to type. The following hematopoietic malignancies are represented: Activated B-cell type, acute lymphoid leukemia, acute myeloid leukemia, B-lymphoblastic leukemia/lymphoma, chronic lymphocytic leukemia, diffuse large B-cell lymphoma and germinal center B-cell type leukemia. Duplicates were removed. SETD2 domains: AWS, associated with SET; SET, Su(var)3-9, enhancer-of-zeste trithorax; PS, post-SET; CC, coiled coil; WW, rsp5-domain; SRI, Set2 Rpb1 interacting. Mutation data were retrieved from cBioPortal (http:/ / www.cbioportal.org) on 27 November 2018.

These observations indicate that heterozygous SETD2 loss, as frequently found in AML patients, accelerates leukemogenesis driven by the MLL-AF9 fusion protein, and perhaps also other oncogenic drivers. In contrast, complete SETD2 loss, as induced by homozygous deletion or near-complete loss-of-function-induced shRNAs or CRISPR/Cas9-mediated mutagenesis significantly delayed disease progression. These seemingly opposing observations imply that homo- versus heterozygous SETD2 loss has significantly different effects on leukemogenesis. As the majority of cancer patients present with heterozygous mutations in SETD2, it is conceivable that SETD2 functions as a haplo-insufficient tumor suppressor, while its homozygous loss impedes disease progression. Such a scenario might be of therapeutic relevance, as complete ablation of SETD2 may preferentially impact leukemia cells harboring heterozygous mutations of SETD2. However, homozygous depletion of SETD2 in T-cells was associated with rapid expansion of the $\gamma \delta$-T-cell population [21]. This indicates that SETD2-dependent effects might be context-specific. Furthermore, it might be important to differentiate between effects that depend on the enzymatic activity of SETD2 (such as H3K36 methylation) and potential other molecular functions of SETD2 in the context of hematopoietic malignancies.

\section{Mechanism of Action of SETD2 in Leukemia and Oncogenesis}

SETD2 has been implicated in a number of cellular processes, many of which are dysregulated in cancer. The relative contribution of SETD2 to these molecular pathways is unclear, and we are only beginning to understand how dysbalanced SETD2 levels affect these processes in the context of a malignant cell. In the following, we will highlight the most important cellular functions of SETD2 and their potential links to leukemia.

\subsection{SETD2-Dependent Regulation of the DNA Damage Response}

The SETD2-dependent H3K36me3 mark is recognized by a number of epigenetic readers which bind this histone modification through chromodomains, such as PWWP (Proline-Tryptophan-Tryptophan-Proline) or Tudor (Figure 2) [3,31]. Loss of SETD2 was associated 
with altered DNA mismatch repair (MMR), as MSH6 (MutS homolog 6), a known component of the MutS $\alpha$ mismatch recognition complex, interacts with the H3K36me3 mark via its PWWP domain. Loss of SETD2-mediated H3K36 methylation as well as loss-of-function mutations of the MSH6 PWWP domain resulted in increased frequencies of spontaneous mutations, which are characteristic for MMR [32]. In line with this, LEDGF (Lens epithelium-derived growth factor, PSIP1), a known interaction partner of MLL and MLL-fusion proteins in acute leukemias utilizes its PWWP domain to bind to H3K36me3 and facilitates DNA-end resection during homologous recombination (HR). In addition, LEDGF was shown to recruit the DNA-damage repair machinery including RAD51, RPA (Replication protein A), and RBBP8 (CtBP-interacting protein, CtIP) to sites of damaged DNA [33,34]. The Tudor domain-containing protein PHF1 (PHD finger protein 1), a member of the Polycomb Repressor 2 (PRC2) complex, interacts with DNA double strand breaks marked with H3K36me3 and is involved in early events of the DNA damage response [31]. In this case, loss of SETD2-mediated H3K36 tri-methylation reduced PHF1 retention at double strand break sites, decreasing the efficiency of repair mechanisms. We found that loss of SETD2 resulted in increased DNA damage and activation of the tumor suppressor p53 and its target gene p21 [30]. Transcriptomic analysis identified enriched expression of DNA-damage-associated gene sets in SETD2-deficient cells. The same phenotype was observed in SETD2-deficient ccRCC cells. In this context, loss of SETD2 resulted in the accumulation of DNA damage and inefficient DNA damage repair. However, this was attributed to the inability of SETD2-deficient cells to efficiently activate p53 [35]. This controversy suggests that while observed phenotypes might be similar, the underlying molecular mechanisms might be tissue- and/or context specific. Knowing that SETD2 is part of a large multi-protein assembly nucleated by MLL-fusion proteins in the context of AML, it might be tempting to speculate that specific activities of SETD2 might be strongly dependent on the protein complex architecture in which it is embedded [30].

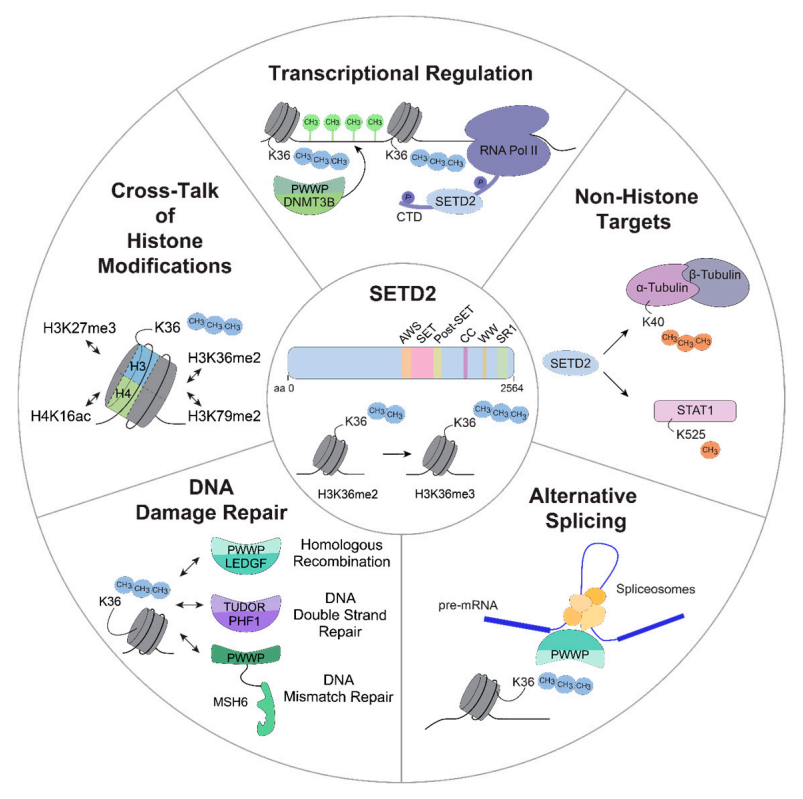

Figure 2. Schematic overview of potential functions of SETD2. SETD2-mediated H3K36 tri-methylation is involved in different cellular processes that are potentially associated with oncogenesis, including transcriptional regulation, methylation of non-histone targets, alternative splicing, DNA damage repair and an extensive crosstalk between $\mathrm{H} 3 \mathrm{~K} 36 \mathrm{me} 3$ and other histone modifications. aa, amino acid; H3K36me2/me3, di-/tri-methylation of lysine 36 on histone H3; RNA Pol II, RNA polymerase II; CTD, carboxy terminal domain; DNMT3B, DNMT3B, DNA (cytosine-5-)-methyltransferase 3 beta; STAT1, signal transducer and activator of transcription 1; LEDGF, PC4 and SFRS1 interacting protein 1 (PSP1); PHF1, PHD finger protein 1; MSH6, MutS $\alpha$ homolog 6; H3K79me2, di-methylation of lysine 79 on histone H3; H3K27me3, tri-methylation of lysine 27 on histone H3; H4K16ac, acetylation of lysine 16 on histone $\mathrm{H} 4$. 


\subsection{Cross-Talk between H3K36me3 and Other Histone Modifications}

The importance of SETD2 in MLL-rearranged AML depends on chromatin targeting of the MLL complex by LEDGF, which interacts with methylated H3K36 via its PWWP domain [36]. Loss of H3K36me3 resulted in compromised chromatin binding of MLL-complexes and deregulation of MLL-fusion target gene expression. Down-regulation of SETD2 resulted in decreased binding of the MLL-AF9 fusion protein to promotors of known MLL target genes, such as Hoxa9 (Homeobox protein Hox-A9) and Meis1 (Homeobox protein Meis1, Myeloid ecotropic viral integration site 1), leading to reduced expression of these genes [30]. In another study, it was shown that loss of SETD2-dependent H3K36me3 induced elevated levels of the ASH1L-dependent H3K36me2 mark in MLL-AF9-expressing AML cells [37]. LEDGF was identified to preferentially bind to ASH1L-dependent H3K36me2-marked regions, thereby recruiting MLL-fusion-containing protein complexes to promoter regions. Thus, in this study, loss of SETD2-dependet H3K36me3 led to elevated levels of H3K36me2, enhancing the recruitment of LEDGF-containing MLL complexes to chromatin [37]. As the functional role of H3K36me3 remains elusive, further work is required to resolve these contradictory results.

Extensive crosstalk between the SETD2-dependent H3K36me3 mark and other histone marks has been reported (Figure 2). A strong correlation exists between the localization of the H3K36me3 mark and the DOT1L (Disruptor of telomere silencing protein 1-like)-dependent H3K79me2 mark in MLL-fusion expressing AML [30,38]. A dual H3K36me3-high-H3K79me2-high signature characterized MLL-target genes. shRNA-mediated knockdown of SETD2 resulted in a reduction of H3K36me3, but also in decreased H3K79me2 levels on MLL-target genes, indicating a strong interdependence of these histone marks. This is in contrast to data by Zhou et al. who found increased global H3K79me2 levels upon loss of Setd2 [12]. This report identified a link between the loss of H3K36me3 and elevated levels of ASH1L, NSD1, NSD2 and NSD3 enzymes responsible for mono- and di-methylation of H3K36. Another study found that loss of SETD2 led to down-regulated expression of the tumor suppressor ASXL1 (Additional Sex Combs-Like protein 1) and an upregulation of ERG, resulting in accelerated leukemia development [39]. Even though the functional consequences of the enriched co-localization of the H3K36me3 and H3K79me2 histone modifications on MLL-target genes remain elusive and controversial, it indicates an important role of this dual signature in the biology of MLL-rearranged leukemia.

Besides the interplay of H3K36me3 with H3K79me2, it was reported that loss of H3K36me3 caused elevated levels of H3K27me3 in sarcomas [40]. This is in contrast to previous findings that suggest that binding of the Tudor domain-containing protein PHF1 to H3K36me3 might sterically preclude the ability of Polycomb complex PRC2 from deposition of the H3K27me3 mark [41]. An interplay between H3K36me3 and H4K16ac was also shown [42]. In this case, H3K36me3 triggers deposition of H4K16ac upon the induction of DNA damage.

Finally, aberrant regulation of epigenetic modulators can alter global gene expression patterns through loss of characteristic histone modifications patterns. Thus, distinct molecular lesions might converge on similar chromatin-based mechanisms to induce misregulation of overlapping gene sets to induce leukemia development. For instance, the gene expression profiles of Setd2-deficient hematopoietic cells were highly similar to global gene expression patterns of cells from Tet2/Dnmt3a-double knockout mice [13].

In summary, despite the presence of a large body of results suggesting the functional importance of epigenetic cross-talk in leukemia development, the mechanistic dependencies between H3K36me3 and other histone modifications remain unclear.

\subsection{SETD2 in Alternative Splicing}

In higher eukaryotes, post-translational modification of histone tails is associated with the regulation of alternative splicing. H3K36me3 was particularly implicated in this process, as intron-containing genes featured higher levels of the H3K36me 3 mark than intron-less genes in mice and humans. In addition, genes containing constitutively included exons feature significantly higher 
H3K36me3 levels than genes with alternatively spliced ones (Figure 2) [43,44]. In agreement with this, shRNA-mediated loss of SETD2 led to reduced levels of exon inclusion in actively transcribed genes and a global switch of splice sites [45]. PWWP-domain containing proteins play a pivotal role in the regulation of this process. For instance, ZMYND11 (Zinc finger MYND-domain containing 11), BRPF1 (Bromodomain and PHD finger-containing 1) and MRG15 (MORF-related gene 15) bind to H3K36me3 and thereby either interact with components of the RNA splicing machinery or influence transcriptional regulation [45-49]. Likewise, the LEDGF-H3K36me3 interaction can modulate RNA splicing through LEDGF-dependent recruitment of spliceosome factors, such as SRSF1 (Serine/arginine-rich splicing factor 1) [50].

Given the strong evidence for a link between SETD2-dependent H3K36me3 and the regulation of alternative splicing, one may speculate that dysregulation of this process might contribute to malignant transformation. To date, however, the involvement of alternative splicing to leukemia development in the context of SETD2 has not been documented.

\subsection{SETD2 in Transcriptional Regulation}

The role of SETD2 in transcriptional elongation has been first described in yeast. The Set2 Rpb1 interacting (SRI) domain of the yeast Set2 protein interacts with the phosphorylated carboxy-terminal domain (CTD) of RNA polymerase II (RNA Pol II) [51]. A similar interaction was confirmed in mammalian cells [52,53]. RNA polymerase II is the integral part of the cellular machinery responsible for transcriptional regulation and interacts with a large number of accessory proteins, including structural proteins, epigenetic regulators, and transcription factors. The RNA Pol II CTD acts as a scaffold for the interaction with factors that regulate initiation, elongation and termination of transcription [54]. As SETD2 interacts with the CTD of elongating RNA Pol II, it was proposed that SETD2-dependent H3K36 tri-methylation across gene bodies prevents spurious intragenic initiation of transcription through the recruitment of a histone deacetylase complex [3]. In addition, SETD2 was demonstrated to play a role in nucleosome reorganization in bodies of transcribed genes after the passage of RNA Pol II machinery [55]. In this context, loss of SETD2 led to intragenic initiation of transcription through abnormal H3K36me3-dependent targeting of the FACT (Facilitates Chromatin Transcription) complex to chromatin, thus decreasing nucleosome occupancy on actively transcribed genes. As a result, down-regulation of SETD2 was associated with the induction of cryptic transcription of a large number of genes [55].

Interestingly, loss of SETD2 caused elevated levels of phosphorylated Serine 2 and Serine 5 in the RNA Pol II CTD, which led to higher levels of chromatin-association of elongating RNA Pol II with the Myc locus in the absence of Setd2 [12]. Furthermore, a link between SETD2 and DNA methylation has been established, as DNMT3B (DNA (cytosine-5)-methyltransferase 3B) binds to the SETD2-dependent H3K36me3 mark via its PWWP-domain (Figure 2) [56]. It was suggested that DNMT3B-mediated methylation of gene bodies protects RNA Pol II from spurious re-initiation of transcription [57].

The global transcriptional effects of H3K36me3 loss might strongly depend on the tissue context. However, several studies found that SETD2 depletion only altered the expression of a small subset of genes rather than causing extensive changes in global gene expression patterns [30,57-60].

Taken together, chromatin binding of protein complexes involved in transcriptional regulation represents another cellular process reliant on SETD2-dependent deposition of H3K36me3. Deregulation of these processes caused by loss or mutation of SETD2 might result in abnormal transcription that is associated with leukemia development.

\subsection{Non-Histone Targets of SETD2}

Interestingly, non-histone targets have been described to be subject to methylation by SETD2 as well (Figure 2). For instance, SETD2 binds and tri-methylates lysine 40 of $\alpha$-Tubulin ( $\alpha$-TubK40me3) on mitotic microtubules [60]. In agreement, loss of SETD2 resulted in genomic instability and cytokinesis defects. This function of SETD2 might have a chromatin-independent impact on DNA damage 
processes and could contribute to the development of malignancies. Furthermore, SETD2 was found to mono-methylate lysine 525 (K525) of the transcription factor STAT1 (Signal transducer and activator of transcription 1) [61]. This modification was shown to enhance Interferon $\alpha$ signaling in hepatocytes, thus reinforcing antiviral immunity.

\section{Therapeutic Opportunities}

The concept of synthetic lethal interactions is of particular interest in cancer therapy, as certain genetic aberrations in cancer cells might expose specific vulnerabilities and resistance mechanisms against cancer drugs. Loss of SETD2 was shown to sensitize ccRCC cells to the PI3K (Phospoinositide 3-kinase) inhibitors TGX221 and AZD6482, thus decreasing the invasive potential of the targeted population [62]. Another study demonstrated that H3K36me3-deficient cancer cells are more sensitive to AZD1775-mediated inhibition of WEE1 kinase [58]. In MLL-fusion expressing leukemia, a combinatorial effect of SETD2 downregulation and pharmacological inhibition of DOT1L was observed [30]. DOT1L is a H3K79me2-specific methyltransferase and a known interactor of MLL-fusion proteins. Small molecule-mediated DOT1L inhibition proved to be a promising strategy in leukemia with MLL-fusion proteins. Targeting DOT1L using the small molecule inhibitor EPZ5676 (Pinometostat) in SETD2-mutated cells potentiated the effects of SETD2-downregulation on growth inhibition, induction of myeloid differentiation and apoptosis. Interestingly, loss of SETD2 and DOT1L inhibition also synergized in the induction of DNA damage [30]. These findings emphasize the need for the development of efficient SETD2 inhibitors. In an effort to characterize inhibitors of the enzymatic activity of SETD2, a drug screening approach revealed N-alkyl Sinefungin derivatives as most potent and selective SETD2 inhibitors when compared to a panel of methyltransferase enzymes [63]. However, given that Sinefungin is not cell permeable, this substance cannot be utilized for functional cellular studies. This raises an urge to identify novel, more potent agents or to find an alternative approach to target SETD2-dependent functions in cancer. The availability of specific small-molecule inhibitors of SETD2 function together with combinatorial approaches might represent a therapeutic strategy for patients suffering from leukemia and other SETD2-dependent malignancies.

\section{Conclusions and Perspectives}

Even though conflicting results have been obtained with regard to the molecular mechanisms involved, it has been unanimously recognized that SETD2 plays a pivotal role in hematopoietic malignancies. Given the diametrically opposed effects of heterozygous versus homozygous loss of SETD2 on leukemia development, it would be beneficial to gain a more profound knowledge about the mutational landscape of SETD2 in hematopoietic cancers. In addition, a global view of the cellular SETD2 protein interaction network is still missing. The identification of SETD2 interaction partners will significantly enhance our knowledge about SETD2 and contribute to the clarification of the role of SETD2 in cancer. Overall, even though an unambiguous mechanism of action of SETD2 in cancer has not been revealed, recent reports have established the role of SETD2 in DNA damage repair, alternative splicing, regulation of transcription as well as a number of epigenetic dependencies. All these processes, when dysregulated, might contribute to the development of malignant diseases.

Author Contributions: A.S., J.E. and F.G. wrote the manuscript. J.E. prepared the illustrations.

Funding: J.E. is supported by grant no. 857935 from the Austrian Research Promotion Agency (FFG). The Grebien laboratory has received funding from the European Research Council under the European Union's Horizon 2020 research and innovation program (grant agreement $n^{\circ} 636855 / \mathrm{StG}$ ).

Conflicts of Interest: The authors declare no conflict of interest.

\section{References}

1. Faber, P.W.; Barnes, G.T.; Srinidhi, J.; Chen, J.; Gusella, J.F.; MacDonald, M.E. Huntingtin interacts with a family of WW domain proteins. Hum. Mol. Genet. 1998, 7, 1463-1474. [CrossRef] [PubMed] 
2. Mao, M.; Fu, G.; Wu, J.S.; Zhang, Q.H.; Zhou, J.; Kan, L.X.; Huang, Q.H.; He, K.L.; Gu, B.W.; Han, Z.G.; et al. Identification of genes expressed in human CD34(+) hematopoietic stem/progenitor cells by expressed sequence tags and efficient full-length cDNA cloning. Proc. Natl. Acad. Sci. USA 1998, 95, 8175-8180. [CrossRef] [PubMed]

3. Wagner, E.J.; Carpenter, P.B. Understanding the language of Lys36 methylation at histone H3. Nat. Rev. Mol. Cell Biol. 2012, 13, 115-126. [CrossRef] [PubMed]

4. Edmunds, J.W.; Mahadevan, L.C.; Clayton, A.L. Dynamic histone H3 methylation during gene induction: HYPB/Setd2 mediates all H3K36 trimethylation. EMBO J. 2008, 27, 406-420. [CrossRef] [PubMed]

5. Yuan, W.; Xie, J.; Long, C.; Erdjument-Bromage, H.; Ding, X.; Zheng, Y.; Tempst, P.; Chen, S.; Zhu, B.; Reinberg, D. Heterogeneous nuclear ribonucleoprotein L is a subunit of human KMT3a/set2 complex required for H3 Lys-36 trimethylation activity in vivo. J. Biol. Chem. 2009, 284, 5701-15707. [CrossRef] [PubMed]

6. Hacker, K.E.; Fahey, C.C.; Shinsky, S.A.; Chiang, Y.J.; DiFiore, J.V.; Jha, D.K.; Vo, A.H.; Shavit, J.A.; Davis, I.J.; Strahl, B.D.; et al. Structure/function analysis of recurrent mutations in SETD2 protein reveals a critical and conserved role for a SET domain residue in maintaining protein stability and histone H3 Lys-36 trimethylation. J. Biol. Chem. 2016, 291, 21283-21295. [CrossRef] [PubMed]

7. McDaniel, S.L.; Strahl, B.D. Shaping the cellular landscape with Set2/SETD2 methylation. Cell. Mol. Life Sci. 2017, 74, 3317-3334. [CrossRef] [PubMed]

8. Rea, S.; Eisenhaber, F.; O'Carroll, D.; Strahl, B.D.; Sun, Z.W.; Schmid, M.; Opravil, S.; Mechtler, K.; Ponting, C.P.; Allis, C.D.; et al. Regulation of chromatin structure by site-specific histone H3 methyltransferases. Nature 2000, 406, 593-599. [CrossRef] [PubMed]

9. Strahl, B.D.; Grant, P.A.; Briggs, S.D.; Sun, Z.W.; Bone, J.R.; Caldwell, J.A.; Mollah, S.; Cook, R.G.; Shabanowitz, J.; Hunt, D.F.; et al. Set2 is a nucleosomal histone H3-selective methyltransferase that mediates transcriptional repression. Mol. Cell. Biol. 2002, 22, 1298-1306. [CrossRef] [PubMed]

10. Li, J.; Duns, G.; Westers, H.; Sijmons, R.; van den Berg, A.; Kok, K. SETD2: An epigenetic modifier with tumor suppressor functionality. Oncotarget 2016, 7, 50719-50734. [CrossRef] [PubMed]

11. Hu, M.; Sun, X.J.; Zhang, Y.L.; Kuang, Y.; Hu, C.Q.; Wu, W.L.; Shen, S.H.; Du, T.T.; Li, H.; He, F.; et al. Histone $\mathrm{H} 3$ lysine 36 methyltransferase Hypb/Setd2 is required for embryonic vascular remodeling. Proc. Natl. Acad. Sci. USA 2010, 107, 2956-2961. [CrossRef] [PubMed]

12. Zhou, Y.; Yan, X.; Feng, X.; Bu, J.; Dong, Y.; Lin, P.; Hayashi, Y.; Huang, R.; Olsson, A.; Andreassen, P.R.; et al. Setd2 regulates quiescence and differentiation of adult hematopoietic stem cells by restricting RNA polymerase II elongation. Haematologica 2018, 103, 1110-1123. [CrossRef] [PubMed]

13. Zhang, Y.-L.; Sun, J.W.; Xie, Y.Y.; Zhou, Y.; Liu, P.; Song, J.C.; Xu, C.H.; Wang, L.; Liu, D.; Xu, A.N.; et al. Setd2 deficiency impairs hematopoietic stem cell self-renewal and causes malignant transformation. Cell Res. 2018, 28, 476-490. [CrossRef] [PubMed]

14. The Cancer Genome Atlas Research Network. Comprehensive molecular characterization of clear cell renal cell carcinoma. Nature 2013, 499, 43-49. [CrossRef] [PubMed]

15. Dalgliesh, G.L.; Furge, K.; Greenman, C.; Chen, L.; Bignell, G.; Butler, A.; Davies, H.; Edkins, S.; Hardy, C.; Latimer, $\mathrm{C}$; et al. Systematic sequencing of renal carcinoma reveals inactivation of histone modifying genes. Nature 2010, 463, 360-363. [CrossRef] [PubMed]

16. Duns, G.; Hofstra, R.M.; Sietzema, J.G.; Hollema, H.; van Duivenbode, I.; Kuik, A.; Giezen, C.; Jan, O.; Bergsma, J.J.; Bijnen, H.; et al. Targeted exome sequencing in clear cell renal cell carcinoma tumors suggests aberrant chromatin regulation as a crucial step in ccRCC development. Hum. Mutat. 2012, 33, 1059-1062. [CrossRef] [PubMed]

17. Fontebasso, A.M.; Liu, X.Y.; Sturm, D.; Jabado, N. Chromatin remodeling defects in pediatric and young adult glioblastoma: A tale of a variant histone 3 tail. Brain Pathol. 2013, 23, 210-216. [CrossRef] [PubMed]

18. Yuan, H.; Li, N.; Fu, D.; Ren, J.; Hui, J.; Peng, J.; Liu, Y.; Qiu, T.; Jiang, M.; Pan, Q.; et al. Histone methyltransferase SETD2 modulates alternative splicing to inhibit intestinal tumorigenesis. J. Clin. Investig. 2017, 127, 1-17. [CrossRef] [PubMed]

19. Zhang, J.; Ding, L.; Holmfeldt, L.; Wu, G.; Heatley, S.L.; Payne-Turner, D.; Easton, J.; Chen, X.; Wang, J.; Rusch, M.; et al. The genetic basis of early T-cell precursor acute lymphoblastic leukaemia. Nature 2012, 481, 157-163. [CrossRef] [PubMed] 
20. Martinelli, G.; Mancini, M.; de Benedittis, C.; Rondoni, M.; Papayannidis, C.; Manfrini, M.; Meggendorfer, M.; Calogero, R.; Guadagnuolo, V.; Fontana, M.C.; et al. SETD2 and histone H3 lysine 36 methylation deficiency in advanced systemic mastocytosis. Leukemia 2018, 32, 139-148. [CrossRef] [PubMed]

21. Moffitt, A.B.; Ondrejka, S.L.; McKinney, M.; Rempel, R.E.; Goodlad, J.R.; Teh, C.H.; Leppa, S.; Mannisto, S.; Kovanen, P.E.; Tse, E.; et al. Enteropathy-associated T cell lymphoma subtypes are characterized by loss of function of SETD2. J. Exp. Med. 2017, 214, 1371-1386. [CrossRef] [PubMed]

22. Parker, H.; Strefford, J.C. The mutational signature of chronic lymphocytic leukemia. Biochem J. 2016, 473, 3725-3740. [CrossRef] [PubMed]

23. Mar, B.G.; Bullinger, L.B.; McLean, K.M.; Grauman, P.V.; Harris, M.H.; Stevenson, K.; Neuberg, D.S.; Sinha, A.U.; Sallan, S.E.; Silverman, L.B.; et al. Mutations in epigenetic regulators including SETD2 are gained during relapse in paediatric acute lymphoblastic leukaemia. Nat. Commun. 2014, 5, 3469. [CrossRef] [PubMed]

24. Mar, B.G.; Chu, S.H.; Kahn, J.D.; Krivtsov, A.V.; Koche, R.; Castellano, C.A.; Kotlier, J.L.; Zon, R.L.; McConkey, M.E.; Chabon, J.; et al. SETD2 alterations impair DNA damage recognition and lead to resistance to chemotherapy in leukemia. Blood 2017, 130, 2631-2641. [CrossRef] [PubMed]

25. Zhu, X.; He, F.; Zeng, H.; Ling, S.; Chen, A.; Wang, Y.; Yan, X.; Wei, W.; Pang, Y.; Cheng, H.; et al. Identification of functional cooperative mutations of SETD2 in human acute leukemia. Nat. Genet. 2014, 46, 287-293. [CrossRef] [PubMed]

26. Hart, T.; Chandrashekhar, M.; Aregger, M.; Steinhart, Z.; Brown, K.R.; MacLeod, G.; Mis, M.; Zimmermann, M.; Fradet-Turcotte, A.; Sun, S.; et al. High-resolution CRISPR screens reveal fitness genes and genotype-specific cancer liabilities. Cell 2015, 163, 1515-1526. [CrossRef] [PubMed]

27. Tzelepis, K.; Koike-Yusa, H.; de Braekeleer, E.; Li, Y.; Metzakopian, E.; Dovey, O.M.; Mupo, A.; Grinkevich, V.; Li, M.; Mazan, M.; et al. A CRISPR dropout screen identifies genetic vulnerabilities and therapeutic targets in acute myeloid leukemia. Cell Rep. 2016, 17, 1193-1205. [CrossRef] [PubMed]

28. Post, Y.; Birsoy, K.; Hughes, N.W.; Krupczak, K.M.; Post, Y.; Wei, J.J.; Lander, E.S.; Sabatini, D.M. Identification and characterization of essential genes in the human genome. Science 2015, 350, 1096-1101.

29. Shi, J.; Wang, E.; Milazzo, J.P.; Wang, Z.; Kinney, J.B.; Vakoc, C.R. Discovery of cancer drug targets by CRISPR-Cas9 screening of protein domains. Nat. Biotechnol. 2015, 33, 661-667. [CrossRef] [PubMed]

30. Skucha, A.; Ebner, J.; Schmöllerl, J.; Roth, M.; Eder, T.; César-Razquin, A.; Stukalov, A.; Vittori, S.; Muhar, M.; Lu, B.; et al. MLL-fusion-driven leukemia requires SETD2 to safeguard genomic integrity. Nat. Commun. 2018, 9, 1983. [CrossRef] [PubMed]

31. Musselman, C.A.; Avvakumov, N.; Watanabe, R.; Abraham, C.G.; Lalonde, M.E.; Hong, Z.; Allen, C.; Roy, S.; Nuñez, J.K.; Nickoloff, J.; et al. Molecular basis for H3K36me3 recognition by the Tudor domain of PHF1. Nat. Struct. Mol. Biol. 2012, 19, 1266-1272. [CrossRef] [PubMed]

32. Li, F.; Mao, G.; Tong, D.; Huang, J.; Gu, L.; Yang, W.; Li, G.M. The histone mark H3K36me3 regulates human DNA mismatch repair through its interaction with MutSalpha. Cell 2013, 153, 590-600. [CrossRef] [PubMed]

33. Daugaard, M.; Baude, A.; Fugger, K.; Povlsen, L.K.; Beck, H.; Sørensen, C.S.; Petersen, N.H.; Sorensen, P.H.; Lukas, C.; Bartek, J.; et al. LEDGF (p75) promotes DNA-end resection and homologous recombination. Nat. Struct. Mol. Biol. 2012, 19, 803-810. [CrossRef] [PubMed]

34. Pfister, S.X.; Ahrabi, S.; Zalmas, L.P.; Sarkar, S.; Aymard, F.; Bachrati, C.Z.; Helleday, T.; Legube, G.; La Thangue, N.B.; Porter, A.C.; et al. SETD2-dependent histone H3K36 trimethylation is required for homologous recombination repair and genome stability. Cell Rep. 2014, 7, 2006-2018. [CrossRef] [PubMed]

35. Carvalho, S.; Vítor, A.C.; Sridhara, S.C.; Martins, F.B.; Raposo, A.C.; Desterro, J.M.; Ferreira, J.; de Almeida, S.F. SETD2 is required for DNA double-strand break repair and activation of the p53-mediated checkpoint. elife 2014, 3, e02482. [CrossRef] [PubMed]

36. Okuda, H.; Kawaguchi, M.; Kanai, A.; Matsui, H.; Kawamura, T.; Inaba, T.; Kitabayashi, I.; Yokoyama, A. MLL fusion proteins link transcriptional coactivators to previously active CpG-rich promoters. Nucleic Acids Res. 2014, 42, 4241-4256. [CrossRef] [PubMed]

37. Zhu, L.; Li, Q.; Wong, S.H.; Huang, M.; Klein, B.J.; Shen, J.; Ikenouye, L.; Onishi, M.; Schneidawind, D.; Buechele, C.; et al. ASH1L links histone H3 lysine 36 dimethylation to MLL leukemia. Cancer Discov. 2016, 6, 770-783. [CrossRef] [PubMed] 
38. Bernt, K.M.; Zhu, N.; Sinha, A.U.; Vempati, S.; Faber, J.; Krivtsov, A.V.; Feng, Z.; Punt, N.; Daigle, A.; Bullinger, L.; et al. MLL-rearranged leukemia is dependent on aberrant H3K79 methylation by DOT1L. Cancer Cell 2011, 20, 66-78. [CrossRef] [PubMed]

39. Bu, J.; Chen, A.; Yan, X.; He, F.; Dong, Y.; Zhou, Y.; He, J.; Zhan, D.; Lin, P.; Hayashi, Y.; et al. SETD2-mediated crosstalk between H3K36me3 and H3K79me2 in MLL-rearranged leukemia. Leukemia 2018, 32, 890-899. [CrossRef] [PubMed]

40. Lu, C.; Jain, S.U.; Hoelper, D.; Bechet, D.; Molden, R.C.; Ran, L.; Murphy, D.; Venneti, S.; Hameed, M.; Pawel, B.R.; et al. Histone H3K36 mutations promote sarcomagenesis through altered histone methylation landscape. Science 2016, 352, 844-849. [CrossRef] [PubMed]

41. Musselman, C.A.; Lalonde, M.-E.; Côté, J.; Kutateladze, T.G. Perceiving the epigenetic landscape through histone readers. Nat. Struct. Mol. Biol. 2012, 19, 1218-1227. [CrossRef] [PubMed]

42. Li, L.; Wang, Y. Cross-talk between the H3K36me3 and H4K16ac histone epigenetic marks in DNA double-strand break repair. J. Biol. Chem. 2017, 292, 11951-11959. [CrossRef] [PubMed]

43. Kolasinska-Zwierz, P.; Down, T.; Latorre, I.; Liu, T.; Liu, X.S.; Ahringer, J. Differential chromatin marking of introns and expressed exons by H3K36me3. Nat. Genet. 2009, 41, 376-381. [CrossRef] [PubMed]

44. De Almeida, S.F.; Grosso, A.R.; Koch, F.; Fenouil, R.; Carvalho, S.; Andrade, J.; Levezinho, H.; Gut, M.; Eick, D.; Gut, I.; et al. Splicing enhances recruitment of methyltransferase HYPB/Setd2 and methylation of histone H3 Lys36. Nat. Struct. Mol. Biol. 2011, 18, 977-983. [CrossRef] [PubMed]

45. Luco, R.F.; Pan, Q.; Tominaga, K.; Blencowe, B.J.; Pereira-Smith, O.M.; Misteli, T. Regulation of alternative splicing by histone modifications. Science 2010, 327, 996-1000. [CrossRef] [PubMed]

46. Zhang, P.; Du, J.; Sun, B.; Dong, X.; Xu, G.; Zhou, J.; Huang, Q.; Liu, Q.; Hao, Q.; Ding, J. Structure of human MRG15 chromo domain and its binding to Lys36-methylated histone H3. Nucleic Acids Res. 2006, 34, 6621-6628. [CrossRef] [PubMed]

47. Wen, H.; Li, Y.; Xi, Y.; Jiang, S.; Stratton, S.; Peng, D.; Tanaka, K.; Ren, Y.; Xia, Z.; Wu, J.; et al. ZMYND11 links histone H3.3K36me3 to transcription elongation and tumour suppression. Nature 2014, 508, $263-268$. [CrossRef] [PubMed]

48. Ullah, M.; Pelletier, N.; Xiao, L.; Zhao, S.P.; Wang, K.; Degerny, C.; Tahmasebi, S.; Cayrou, C.; Doyon, Y.; Goh, S.L.; et al. Molecular Architecture of quartet MOZ/MORF histone acetyltransferase complexes. Mol. Cell. Biol. 2008, 28, 6828-6843. [CrossRef] [PubMed]

49. Vezzoli, A.; Bonadies, N.; Allen, M.D.; Freund, S.M.; Santiveri, C.M.; Kvinlaug, B.T.; Huntly, B.J.; Göttgens, B.; Bycroft, M. Molecular basis of histone H3K36me3 recognition by the PWWP domain of Brpf1. Nat. Struct. Mol. Biol. 2010, 17, 617-619. [CrossRef] [PubMed]

50. Pradeepa, M.M.; Sutherland, H.G.; Ule, J.; Grimes, G.R.; Bickmore, W.A. Psip1/Ledgf p52 binds methylated histone H3K36 and splicing factors and contributes to the regulation of alternative splicing. PLoS Genet. 2012, 8, e1002717. [CrossRef] [PubMed]

51. Li, J.; Moazed, D.; Gygi, S.P. Association of the histone methyltransferase Set2 with RNA polymerase II plays a role in transcription elongation. J. Biol. Chem. 2002, 277, 49383-49388. [CrossRef] [PubMed]

52. Li, M.; Phatnani, H.P.; Guan, Z.; Sage, H.; Greenleaf, A.L.; Zhou, P. Solution structure of the Set2-Rpb1 interacting domain of human Set2 and its interaction with the hyperphosphorylated C-terminal domain of Rpb1. Proc. Natl. Acad. Sci. USA 2005, 102, 17636-17641. [CrossRef] [PubMed]

53. Krogan, N.J.; Kim, M.; Tong, A.; Golshani, A.; Cagney, G.; Canadien, V.; Richards, D.P.; Beattie, B.K.; Emili, A.; Boone, C.; et al. Methylation of histone $\mathrm{H} 3$ by Set2 in Saccharomyces cerevisiae is linked to transcriptional elongation by RNA polymerase II. Mol. Cell. Biol. 2003, 23, 4207-4218. [CrossRef] [PubMed]

54. Hahn, S. Structure and mechanism of the RNA polymerase II transcription machinery. Nat. Struct. Mol. Biol. 2004, 11, 394-403. [CrossRef] [PubMed]

55. Carvalho, S.; Raposo, A.C.; Martins, F.B.; Grosso, A.R.; Sridhara, S.C.; Rino, J.; Carmo-Fonseca, M.; de Almeida, S.F. Histone methyltransferase SETD2 coordinates FACT recruitment with nucleosome dynamics during transcription. Nucleic Acids Res. 2013, 41, 2881-2893. [CrossRef] [PubMed]

56. Baubec, T.; Colombo, D.F.; Wirbelauer, C.; Schmidt, J.; Burger, L.; Krebs, A.R.; Akalin, A.; Schübeler, D. Genomic profiling of DNA methyltransferases reveals a role for DNMT3B in genic methylation. Nature 2015, 520, 243-247. [CrossRef] [PubMed] 
57. Neri, F.; Rapelli, S.; Krepelova, A.; Incarnato, D.; Parlato, C.; Basile, G.; Maldotti, M.; Anselmi, F.; Oliviero, S. Intragenic DNA methylation prevents spurious transcription initiation. Nature 2017, 543, 72-77. [CrossRef] [PubMed]

58. Pfister, S.X.; Markkanen, E.; Jiang, Y.; Sarkar, S.; Woodcock, M.; Orlando, G.; Mavrommati, I.; Pai, C.-C.; Zalmas, L.-P.; Drobnitzky, N.; et al. Inhibiting WEE1 selectively kills histone H3K36me3-deficient cancers by dNTP starvation. Cancer Cell 2015, 28, 557-568. [CrossRef] [PubMed]

59. Kanu, N.; Grönroos, E.; Martinez, P.; Burrell, R.A.; Yi Goh, X.; Bartkova, J.; Maya-Mendoza, A.; Mistrík, M.; Rowan, A.J.; Patel, H.; et al. SETD2 loss-of-function promotes renal cancer branched evolution through replication stress and impaired DNA repair. Oncogene 2015, 34, 5699-5708. [CrossRef] [PubMed]

60. Park, I.Y.; Powell, R.T.; Tripathi, D.N.; Dere, R.; Ho, T.H.; Blasius, T.L.; Chiang, Y.C.; Davis, I.J.; Fahey, C.C.; Hacker, K.E.; et al. Dual chromatin and cytoskeletal remodeling by SETD2. Cell 2016, 166, 950-962. [CrossRef] [PubMed]

61. Chen, K.; Liu, J.; Liu, S.; Xia, M.; Zhang, X.; Han, D.; Jiang, Y.; Wang, C.; Cao, X. Methyltransferase SETD2-mediated methylation of STAT1 is critical for interferon antiviral activity. Cell 2017, 170, 492-506. [CrossRef] [PubMed]

62. Feng, C.; Sun, Y.; Ding, G.; Wu, Z.; Jiang, H.; Wang, L.; Ding, Q.; Wen, H. PI3K $\beta$ inhibitor TGX221 selectively inhibits renal cell carcinoma cells with both VHL and SETD2 mutations and links multiple pathways. Sci. Rep. 2015, 5, 9465. [CrossRef] [PubMed]

63. Zheng, W.; Ibáñez, G.; Wu, H.; Blum, G.; Zeng, H.; Dong, A.; Li, F.; Hajian, T.; Allali-Hassani, A.; Amaya, M.F.; et al. Sinefungin derivatives as inhibitors and structure probes of protein lysine methyltransferase SETD2. J. Am. Chem. Soc. 2012, 134, 18004-18014. [CrossRef] [PubMed]

(C) 2019 by the authors. Licensee MDPI, Basel, Switzerland. This article is an open access article distributed under the terms and conditions of the Creative Commons Attribution (CC BY) license (http:/ / creativecommons.org/licenses/by/4.0/). 